P0-0918 THE POSTOPERATIVE OUTCOME OF PAEDIATRIC SURGICAL PATIENTS DURING 2008-2013. IN BOSNIA AND HERZEGOVINA

${ }^{1}$ A Jonuzi, ${ }^{2} S$ Dinarevic-Mesihovic, ${ }^{2} \mathrm{~F}$ Jonuzi. ${ }^{1}$ Pediatric Surgery, Clinical Center University in Sarajevo, Sarajevo, Bosnia and Herzegovina; ${ }^{2}$ Paediatric Clinic, Clinical Center University in Sarajevo, Sarajevo, Bosnia and Herzegovina

\subsection{6/archdischild-2014-307384.1539}

Introduction With improvement in postoperative care of surgical, cardiac, neurosurgical and other surgical paediatric patients, better outcome has been proven. Pts after surgery were transffered at Paediatric, Neonatal Intensive and Postintensive Cardiac Care Units at Paediatric clinic.

Methods Retrospective evaluation of patients treated during January 2008-November 2013 period was performed. Patients were classified according to: sex, age, type of disease, total and specific mortality.

Results Study included 468 postoperative patients. Paediatric surgery was represented with $64.9 \%$. Cardiac surgery $21.7 \%$, Neurosurgery $8.9 \%$, and others $4.2 \%$. Morbidity structure of surgical patients was: EA 12 (3.9\%), gastroschisis 8 (2.6\%), omphalocele 5 (1.6\%), CDH 6 (2\%), NEC 15(4.9\%), urogenital procedures 75 (24.7\%), GI tract-inflammations 41 (13.5\%), congenital megacolon 9 (3\%), bowel obstruction 72 (23.7\%), tumour procedures 44 (14.5\%). Cardiac structure: ToF 28 (27.4\%), VSD 24 (23.5\%), ASD 14(13.7\%), complex CHD 14 (13.7\%), AVSD-a 8 (7.8\%), TGA 3 (2.9\%), AP stenosis 3 (2.9\%), DAP 8 (7.8\%). Hydrocephalus was present in 26 patients $(66.6 \%)$. Total surgical mortality: $14.5 \%$, specific paediatric surgery mortality was: $12.5 \%$, cardiac: $16.6 \%$, neurosurgical: $23.8 \%$, others $15 \%$. In morbidity structure NEC was present in $26.3 \%$, survival of $34 \%, \mathrm{CDH} 50 \%$, gastroschisis $62.5 \%$ and EA $75 \%$. Survival post cardiac surgery was $100 \%$ for DAP, pulmonary artery stenosis 96\%, VSD 93\%, ASD 82\%, ToF $75 \%$, AVSD $57 \%$, 34\% for TGA. Survival of hydrocephalus with comorbidity was $20 \%$.

Conclusion Morbidity and mortality of postoperative care patients points for further improvement and development of children health care, having in mind that requres team's work.

\section{PO-0919 TISSUE EXPANSION TECHNIQUE FOR TREATMENT OF CONGENITAL HAND SYNDACTYLY}

D Kravljanac, R Simic. Plastic and Reconstructive Surgery, Institute for Mother and Child Healthcare Belgrade Serbia, Belgrade, Serbia

\subsection{6/archdischild-2014-307384.1540}

Background and aim Syndactyly is one of the most common congenital anomaly of the hand in children. A great number of syndactyly release techniques have been described over last two centuries. The aim of our study is outcome assessment of congenital syndactyly surgery using temporarily tissue expansion without skin grafts.

Methods Our study included children with congenital hand syndactyly treated by operative technique with temporarily tissue expansion of the dorsal hand skin and local flaps, without skin grafting in period from 2008-2013. In all cases surgery was performed under general anaesthesia. According to the Weber descriptive method functional outcome at the end of follow-up period was categorised as good, fair and bad.

Results Twenty children (13 males, 7 females) aged from 6 months to 6 years (mean 23 months) were operated by described technique. Fourteen cases had complete, while six had incomplete syndactyly mostly involving the third web. Associated anomalies were diagnosed in twelve patients. Follow up period ranged from 6 months to 4 years. Functional results were: good in twelve patients, fair in seven and bad in one case.

Conclusion Surgical procedure with temporarily tissue expansion of the dorsal hand skin and local flaps, without skin grafting is effective method of congenital syndactyly treatment in children with good functional and aesthetic results.

\section{PO-0920 WITHDRAWN}

\section{PO-0921 RECURRENT INGUINAL HERNIAS : THINK METABOLIC !}

${ }^{1} \mathrm{~N}$ Boutrid, ${ }^{1} \mathrm{H}$ Rahmoune, ${ }^{2} \mathrm{M}$ Ketfi, ${ }^{2} \mathrm{~B}$ Bioud. ${ }^{1}$ Pediatrics, Child and Mother Hospital, Setif, Algeria; ${ }^{2}$ Pediatric Surgery, Child and Mother Hospital, Setif, Algeria

\subsection{6/archdischild-2014-307384.1541}

Introduction There is little available evidence on the differential etiologic diagnosis of recurrent inguinal hernia, particularly if the original causative disorder is a storage disease...

Material and methods The examination of un affected boy, at first consulting in surgery for a recurrent inguinal hernia, reveals in addition a marked dysmorphism with a peculiar umbilical hernia; uncommon for a 23 months infant... Careful palpation notes a mild hepato-splenomegaly; rising the suspicion of a Mucopolysaccharidosis -MPS-

Prompt Dry Blood Test for enzymatic essay, combined to urinary glycosaminoglycans - GAG- collection and molecular genetic testing, confirm the diagnosis of MPS 2 - Hunter disease.

Immediate enzyme replacement therapy is ordered, as well as referring to haematology consultancy for a rapid bone marrow transplant

Management is differed after ERT and a possible bone marrow transplant; with a special attention to the anaesthetic dangers for such a condition.

Results and discussion Mucopolysaccharidosis type II (MPS II; also known as Hunter syndrome) is an X-linked multisystem disorder characterised by glycosaminoglycans (GAG) accumulation.

The latter gives an hyperlaxity aspect to the mucocutaneous tissues leading to such recurrent hernias

Surgeons ought to be aware of this peculiar presentation of attenuated mucopolysaccharidosis, in time of onset and types of clinical related features.

Conclusion Recurrent inguinal hernias, specially if associated to dysmorphic features and visceromegaly, should be promptly managed as a probable metabolic - lysosomal storage disease, chiefly a mucopolysaccharidosis. 